\title{
Emilio Modena (Hrsg.): Leidenschaften. Paul Parin zum 90. Geburtstag Berlin (Edition Freitag)
}

Roland Kaufhold (Köln)

Der Psychoanalytiker und Schriftsteller Paul Parin blickt auf ein außergewöhnlich langes und produktives Leben zurück. Für sein die gewohnten wissenschaftlichen Disziplinen und Abgrenzungen überschreitendes Wirken ist er in den letzten Jahren vielfältig ausgezeichnet worden. Im September 2006 wurde Paul Parin, dieser liebenswerte, leidenschaftlich-skeptische Menschenkenner, 90 Jahre alt.

Anlässlich seines 90. Geburtstages führte das PSZ eine wissenschaftliche Tagung durch. Emilio Modena hat nun diese Festvorträge in einem kleinen, mit «Leidenschaften» betitelten Buch versammelt, gemeinsam mit einigen weiteren, aus Parins breitgestreutem Freundeskreis stammenden Beiträgen. Diese schwanken zwischen wissenschaftlicher Analyse, biografischen Interpretationen, sowie stark persönlich getönten Beschreibungen der eigenen Prägungen durch Paul Parins Wirken.

Der Prophet im eigenen Land gilt bekanntlich verschiedentlich nicht viel. Es fällt sehr viel leichter, jemanden zu preisen und zu ehren, der weit weg ist. HorstEberhard Richter, der seit über 45 Jahren in der kleinen Universitätsstadt Gießen wirkt, musste dies erleben. Erst nach einem jahrelangen, kleinkarierten Streit der dortigen Lokalpolitiker wollte man dem überzeugten Pazifisten sein gesellschaftskritisches, pazifistisches Engagement «verzeihen». Im Dezember 2007 wurde H.-E. Richter endlich zum Gießener Ehrenbürger ernannt.

Emilio Modena dürfte vergleichbare Erfahrungen im Hinterkopf gehabt haben. In seinem dem Band vorangestellten Grußwort formuliert er demgemäß, dass nicht nur die Psychoanalyse und seine zahlreichen Schüler und Kollegen Paul Parin viel verdankten, sondern «auch die Stadt Zürich»:» Seit dem Zweiten Weltkrieg war Paul Parin ein Vorbild als engagierter Zeitgenosse und Antifaschist. 1944/45 ging er mit einer Ärztemission der Centrale Sanitaire Suisse nach Montenegro, um den Tito-Partisanen zu helfen, und 1968 stand er im Zürcher Manifest den bewegten Zürcher Jugendlichen zur Seite. Er hat in zahllosen Verlautbarungen, Interviews und Zeitungsartikeln die kritische Intelligenz und das soziale Gewissen vertreten und uns zudem immer wieder als begnadeter Geschichtenerzähler und 
Schriftsteller bereichert» (8). An diesem bereits gesteckten Rahmen orientieren sich die nachfolgenden zehn Beiträge über Paul Parin.

Die Schriftstellerin Christa Wolf fühlt sich Parins Schreiben sehr verbunden. Als Parin 1992 auf ihren Vorschlag hin für sein literarisches Erstlingswerk den Erich Fried Preis verliehen bekam, hielt sie die Laudatio. Als einen «moralischen Anarchisten» hatte sie ihn seinerzeit bezeichnet - und diese Formulierung wandelt sie nun in ihrem anrührenden Beitrag in «Ein fröhlicher Anarchist» (15) um. Wolf erinnert an ihre erste Begegnung mit Parin, ihre Lektüre seines literarischen Afrikabuches "Zu viele Teufel im Land» (1984). Dieses vom Gestus der Neugierde, der emotionalen Offenheit und Unerschrockenheit getragene Werk stachelte ihre eigene Neugierde an, ermutigte sie aber auch zu einer unsentimentalen Illusionslosigkeit: «Heiter, freundlich, souverän das Leben genießen, von sich selbst und von den Mitstreitern eine moralische Anstrengung verlangen, ohne sich zu verkrampfen (...) scheinbar erfolglos bleib(en) und doch nicht bitter werden, sondern der Aufklärung verpflichtet bleiben» (16) - diese Einstellung Parins zum Leben hat Christa Wolf tief beeindruckt.

In vergleichbarem, von Ermutigung geprägtem Tenor sind die vor allem an Parins literarischen Werken orientierten Beiträge von Karl-Markus Gauß: «Das Talent zum Glück», von Stefan Zweifel: «Mitten im Fluss der Triebe» sowie vom Schriftsteller Urs Widmer: «Paul Parin, der Geschichtenerzähler» gehalten. Widmer verdeutlicht, dass bereits Parins frühen ethnopsychoanalytischen Werke von einer sie prägenden literarischen Qualität gekennzeichnet waren. Der Übergang vom wissenschaftlichen zum schriftstellerischen Schreiben war ein gleitender Prozess, war bereits in seinen Erstlingswerken angelegt: «Dass der Arzt und Wissenschaftler sich in einen Erzähler verwandelte, überraschte gewiss manche. Jetzt allerdings, in der Rückschau, sieht sein Schreibweg logisch und notwendig aus. Wenn es so etwas wie einen 'geborenen Erzähler` gibt, dann ist Paul Parin einer. Er kann gar nicht anders als erzählen» (96). Parins Neugier, seine Abenteuerlust, wurden zur Quelle seiner literarischen und wissenschaftlichen Produktivität - in Slowenien, in Jugoslawien, in Afrika und auch in Zürich.

Helga Rabenstein-Moser führt diese Analyse in ihrem längeren, biografisch geprägten Beitrag «Die Orte des Paul Parin: Aufenthalt und Bewegung» fort. Sie geht von Parins Schlüsseltext «Kurzer Aufenthalt in Triest oder Koordinaten der Psychoanalyse» (1988) aus, auf welchen in diesem Buch mehrfach zustimmend verwiesen wird. Hierin schilderte Parin in verdichteterWeise seinen biografischen Übergang von seiner Partisanentätigkeit im Jugoslawien der Jahre 1944/45 hin zu seiner analytischen Ausbildung in Zürich. Sie erinnert an Parins Herkunft aus 
einem multikulturellen, privilegierten jüdischen Elternhaus, an seine Jugend im Gut Novikloster in Slowenien, an welche er sich in seinem literarischen Erstlingswerk «Untrügliche Zeichen von Veränderung» (1980) erinnert hat. Im multikulturellen Triest liegen seine Großeltern auf dem jüdischen Friedhof begraben, diese Stadt fasziniert ihn - und doch vermag er sehr nüchtern über sie zu erzählen. Triest wurde zum Wendepunkt in seinem Leben, zu welchem er 40 Jahre später wieder zurückgeht, und von dem er - der nun Fremde - sich doch wieder zu lösen vermag. Es ist ein passagerer und doch ein bleibender Ort - aufgehoben in seinen Erzählungen. Analyse, Anteilnahme, schmerzhafte Erinnerungen an von den Deutschen ermordete Verwandte, Landschaftsbeschreibungen, poetische Betrachtungen, analytische Deutungen und historische Fakten - alles gehört bei Paul Parin zusammen. Auf diese Weise weckt Parin die Phantasie und Leidenschaft seiner Leser - wie ihm dies auch später, in seinem Partisanenbuch «Es ist Krieg und wir gehen hin» (1991) und in seinem erregend-verstörendem Alterswerk «Die Leidenschaft des Jägers» (2003) gelungen ist. Seine literarischen und seine wissenschaftlichen Schriften gehören untrennbar zusammen, sind der Kern seines internationalen Erfolges.

Christian Maier analysiert in «Die Leidenschaft des Psychoanalytikers» Parins gesellschaftskritisch-psychoanalytischen Werke, die aus einer mehrere Jahrzehnte überdauernden analytischen Tätigkeit erwachsen sind. Erinnert sei an seine Aufsatzsammlungen «Subjekt im Widerspruch» (1978) und «Widerspruch im Subjekt» (1986, zusammen mit Goldy Parin-Matthèy). Der Verzicht auf emotionale Geborgenheit, die «Grundhaltung, jederzeit alles in Frage zu stellen» (52) bilde für Parin die Voraussetzung einer wie auch immer theoretisch begründeten Psychotherapie. Diese Grundhaltung dürfe jedoch nicht mit Neutralität verwechselt werden, weil der Psychoanalytiker «stets leidenschaftlich auf Seiten des Unterdrückten stehe»(54). Die Leidenschaft des Psychoanalytikers münde in eine unendliche Analyse, und Paul Parin habe dies wie kein Zweiter überzeugend gelebt.

Maya Nadig, die in Bremen an der Universität Ethnopsychoanalyse lehrt, erinnert in «Die Ethnopsychoanalyse von Paul Parin - komplex, menschlich und in einem unkonventionellen Sinne revolutionär» an Paul Parins frühen ethnopsychoanalytischen Studien. Sieben mal waren die Parins und die Morgenthalers, von der Abenteuerlust sowie einem unstillbaren Wissensdurst angetrieben, in den 1950er und 1960er Jahren zu jeweils mehrmonatigen Forschungsreisen nach Afrika aufgebrochen, hatten «ausgesprochen pionierhaft (...) die psychoanalytische Gesprächstechnik zum ersten Mal direkt in das ethnologische Feld eingeführt» 
(64), hierbei immer die «Grenzen zwischen liebevoller Idealisierung und sachlichem Hinschauen» (64 f.) beachtend. Die wissenschaftliche Methode selbst wurde hierbei in ihrem prozesshaften Gang herausgearbeitet, für Nadig ein wegweisender Paradigmenwechsel. Die Vermittlung dieses komplexen, höchst anspruchsvollen Verfahrens an der Universität wird als ein schwieriger, aber dennoch möglicher interdisziplinärer Weg beschrieben. Nadig endet mit der Bemerkung: «In dieser Menschlichkeit der Methode liegt auch ihr politisches und revolutionäres Potential» (74).

Am Ende dieses durchgängig gut geschriebenen Bandes stehen zwei längere, theoretisch orientierte Beiträge von Ludger Lütkehaus über «Die Leidenschaft des Jägers» - in welchem er den Fokus auf Parins Spätwerk (2003) legt - , sowie von Mario Erdheim über Parins Beiträge zur Ethnopsychoanalyse und zur Psychoanalyse des Jugendalters. 